\title{
Impact of statins and angiotensin- converting enzyme inhibitors on mortality of subjects hospitalised with pneumonia
}

\author{
E.M. Mortensen*\#, M.J. Pugh*,\#, L.A. Copeland*, , M.I. Restrepo*,+, J.E. Cornell*, \\ A. Anzueto ${ }^{+}$and J.A. Pugh*,\#
}

ABSTRACT: Recent studies suggest that statins and angiotensin-converting enzyme (ACE) inhibitors may have beneficial effects for some types of infections. The present study aimed to examine the association of outpatient use of these medications on 30-day mortality for subjects aged $>65$ yrs and hospitalised with community-acquired pneumonia.

A retrospective national cohort study was conducted using the Department of Veterans Affairs administrative data including subjects aged $\geqslant 65$ yrs hospitalised with community-acquired pneumonia, and having $\geqslant 1 \mathrm{yr}$ of prior Veterans Affairs outpatient care.

In total, 8,652 subjects were identified with a mean age of 75 yrs, 98.6\% were male, and $9.9 \%$ of subjects died within 30 days of presentation. In this cohort, $18.1 \%$ of subjects were using statins and $33.9 \%$ were using ACE inhibitors. After adjusting for potential confounders, current statin use (odds ratio (OR) 0.54 , 95\% confidence interval $(\mathrm{Cl})$ 0.42-0.70) and ACE inhibitor use (OR 0.80, 95\% $\mathrm{Cl}$ 0.68-0.89) were significantly associated with decreased 30 -day mortality.

Use of statins and angiotensin-converting enzyme inhibitors prior to admission is associated with decreased mortality in subjects hospitalised with community-acquired pneumonia. Randomised controlled trials are needed to examine whether the use of these medications in patients hospitalised with community-acquired pneumonia may be beneficial.

KEYWORDS: Angiotensin-converting enzyme inhibitor, 3-hydroxl-3-methyl-glutaryl-CoA reductase inhibitor, mortality, pneumonia

$\mathbf{T}$ ogether, pneumonia and influenza are the seventh leading cause of death and the leading causes of infectious death in the USA [1]. For those aged $>65 \mathrm{yrs}$, the rate of hospitalisations and the number of deaths due to pneumonia are increasing, even though the rate is decreasing for all other age groups [2]. Although mortality due to community-acquired pneumonia (CAP) decreased significantly with the introduction of antibiotics in the 1950s, since that time mortality has been stable but hospitalisations are increasing [2]. Despite this, only a few new classes of antibiotics have been added to the armamentarium for treating CAP in the last 20 yrs and only one new class of medication (drotrecogin- $\alpha$ ) has been added [3].

Cytokines play an important role in the host defence mechanisms for subjects with CAP but under certain conditions, may lead to septic shock or acute respiratory distress syndrome [4-6]. Recent studies have demonstrated that
3-hydroxy-3-methyl-glutaryl-CoA reductase inhibitors (statins) and angiotensin converting enzyme (ACE) inhibitors have significant immunomodulatory effects and reduce systemic cytokine levels [7-11]. In addition, several studies have demonstrated that for subjects hospitalised with bacteraemia, diabetic lower extremity infections or CAP, those taking statins or ACE inhibitors at presentation had significantly decreased odds of death [12-15] or decreased rates of severe sepsis [16, 17]. However, other studies have shown either no association with mortality or increased mortality with the use of these medications in subjects with infectious diseases [12, 18, 19].

The present study aimed to assess the association of the use of statins and ACE inhibitors on 30-day mortality for subjects aged $>65$ yrs and hospitalised with CAP after adjusting for other potential confounders using the administrative databases of the Department of Veterans Affairs (VA). It
AFFILIATIONS

*VERDICT,

"Divisions of General Internal Medicine,

+Pulmonary and Critical Care Medicine, and

"Psychiatry, University of Texas Health Science Center at San Antonio, San Antonio, TX, USA.

\section{CORRESPONDENCE}

E.M. Mortensen, VERDICT, ALMD/ UTHSCSA, Ambulatory Care (11C6), 7400 Merton Minter Boulevard, San Antonio, TX 78229, USA

Fax: 12105674423

E-mail: mortensene@uthscsa.edu

Received:

December 112006

Accepted after revision:

September 232007

\section{SUPPORT STATEMENT}

E.M. Mortensen was supported by a Dept of Veterans Affairs Veterans Integrated Service Network 17 new faculty grant and a Howard Hughes Medical Institute faculty start-up grant (00378-001). The Dept of Veterans Affairs supported J.A. Pugh (grants REA 05-129 and RCD 04297) and L.A. Copeland (MREP 05145). M.J. Pugh received funding from the Dept of Veterans Affairs (IIR 02-076 and MREP 02-267). The study is the result of work supported with resources and the use of facilities at the South Texas Veterans Health Care System (San Antonio, TX, USA). The funding agencies had no role in conducting the study or role in the preparation, review or approval of the manuscript.

STATEMENT OF INTEREST None declared.

European Respiratory Journal Print ISSN 0903-1936 Online ISSN 1399-3003 
also aimed to examine the impact of the prior statin and ACE inhibitor use on mortality for subjects hospitalised with pneumonia during the influenza season.

\section{METHODS}

The current study was conducted with VA in-patient and outpatient administrative data, which was collected as part of a larger study of inappropriate prescribing practices in the elderly [20]. The Institutional Review Board of the University of Texas Health Science Center at San Antonio (TX, USA) classified the present study as exempt.

\section{Inclusion and exclusion criteria}

Subjects were included in the present study if they: 1) were aged $\geqslant 65$ yrs on October 1,$1999 ; 2$ ) had at least one outpatient clinic visit during fiscal year (FY) 1999 (October 1, 1998September 30, 1999); 3) were hospitalised during FY 2000 with a primary discharge diagnosis of pneumonia or influenza (International Classification of Disease (ICD)-9 codes 480.0483.99 or 485-487); and 4) had received at least one active and filled medication within 90 days of admission. Previous research has validated the use of ICD-9 codes for identifying cases of CAP [21-23]. Subjects with a history of HIV or who received chemotherapeutic agents within 90 days of presentation were excluded. If a subject was admitted more than once during the study period due to pneumonia, only the first hospitalisation was included.

\section{Data}

The present study used data from the National Patient Care Database at the Austin Automation Center (Austin, TX, USA), pharmacy data from the VA Pharmacy Benefit Management group, and vital status data from the Beneficiary Identification Records Locator Subsystem death file and in-patient portion of the National Patient Care Database.

Demographic information (age, sex, race) was obtained from inpatient and outpatient data. Missing race data were supplemented using self-reported race data from the 1999 Large Health Survey of Veterans [24], a nationally representative survey of VA enrolees (July 1, 1999-January 1, 2000). Race categories included white, black, Hispanic and other/unknown. In addition, information on the VA means test was utilised as a surrogate for income and use of geriatric clinics in the preceding year as a potential indicator of patient frailty.

Comorbid conditions were obtained from in-patient and outpatient administrative data. Charlson's comorbidity score was used to assign a comorbidity score for pre-existing comorbid conditions [25, 26]. Charlson's comorbidity score is based on 19 comorbid conditions, each of which has an associated prognostic weight ranging 1-6. Age was not included in the Charlson score.

Pharmacy data were obtained from the Pharmacy Benefits Management group databases. Subjects were considered current users of a given medication if they had enough tablets to last until the date of hospitalisation, assuming an $80 \%$ compliance rate. Medications classified as statins were atorvastatin, cerivastatin, fluvastatin, lovastatin, pravastatin and simvastatin. Medications classified as ACE inhibitors were benazepril, captopril, enalapril, fosinopril, lisinopril, moexipril, quinapril and ramipril. To further control for potential confounding by medications, a count of unique drugs in each of the following classes per patient was calculated for drugs refilled / filled within 90 days of presentation: cardiac medications (excluding statins, ACE inhibitors and nonstatin lipid-lowering agents) and diabetic medications. A dichotomised variable was created for corticosteroid use. Previous research has demonstrated that using the count of these medication classes is preferable to adjusting for the individual medications [27].

In addition, the current authors created a category of nonstatin lipid-lowering agents (e.g. niacin, bile acid sequestrants and fibric acid derivatives) filled within 90 days of hospital presentation so as to examine confounding in the models.

\section{Outcome}

A 30-day mortality was used as the primary outcome for the present study. Previous research has demonstrated that 30-day mortality is primarily due to CAP rather than other coexisting comorbid conditions [28]. Mortality was assessed using the Beneficiary Identification Records Locator Subsystem and the National Patient Care Database. Previous studies have demonstrated that after 1972, this methodology had a sensitivity of $\sim 96 \%$ for veterans' deaths [29].

\section{Sample size}

For the sample size calculations the current authors assumed: 1) a $15 \%$ overall utilisation of statins and ACE inhibitors; 2) that subjects not taking statins had 2.8 times increased odds, and for ACE inhibitors 2.3 times increased odds, of 30-day mortality based on previous studies [14, 15]; 3) that sample sizes were further boosted by a factor of $\leqslant 1.2$ to account for the random effects; 4 ) the squared multiple correlation among the covariates in the models was $\leqslant 0.40$; and 5) the type I error and power were set at 0.05 and 0.90 , respectively. Therefore, it was calculated that, overall, 4,256 subjects were needed in the statin cohort and 3,620 in the ACE inhibitor cohort to have $90 \%$ probability to detect a significant mortality difference at 30 days.

\section{Statistical analyses}

Bivariate statistics were used to test the association of sociodemographic and clinical characteristics with all-cause 30-day mortality. Categorical variables were analysed using the Chisquared test and continuous variables were analysed using a paired t-test.

A propensity score technique was used to balance covariates associated with medication use between groups [30-32] Separate propensity scores were created for statins, ACE inhibitors and, to examine potential confounding, nonstatin lipid-lowering medications. The propensity score was derived from a logistic regression model. Variables were included in the propensity score if previous research demonstrated a relationship between a variable and pneumonia-related mortality $[33,34]$, if it was hypothesised that it may be related to prescription of the medications, or if the variable was significantly associated $(p \geqslant 0.05)$ with 30 -day mortality or prescription of ACE inhibitors and/or statins in the bivariate analyses. The covariates included in the propensity score models were age, sex, race, being married, VA means test, classes of medications and the Charlson composite score 
(without age). Classes of medications included cardiac medications (excluding ACE inhibitors, statin and nonstatin lipid-lowering medications), diabetic medications and corticosteroids. An ordered categorical variable was then created based on a quintile stratification of the propensity score to include the Cox and regression models.

To analyse time-to-death (starting at the time of hospital admission) for subjects by medication use (statin or ACE inhibitor), Cox proportional hazard models were used to estimate and graph the baseline survivor functions after adjusting for the respective propensity score.

The primary analysis employed generalised linear mixed-effect models, with patient's hospital as a random effect, using the Stata GLLAMM program [35]. Separate models were created with either ACE inhibitors or statin use as the independent variables of interest and adjusting for the appropriate propensity score. In addition, to examine the impact of the medications of interest on pneumonia secondary to influenza, the population was split depending on whether the patient was hospitalised during a period in which influenza was endemic. Those who were admitted between October 1, 1999 and March 31, 2000 (which, according to the Centers for Disease Control and Prevention, was the period with almost all of the influenza cases during the study period [36]) were considered to be during the influenza season, while those hospitalised during other periods were not considered to be related to influenza. Both of the generalised linear mixed-effect models were repeated to examine whether statins or ACE inhibitors were associated with decreased mortality for those hospitalised during the influenza season.

Similar methodology was used to examine the association of nonstatin lipid-lowering drugs with 30-day mortality after adjusting for potential confounders using the appropriate propensity scores. It was hypothesised that nonstatin lipidlowering medications would not be associated with mortality since they have not been demonstrated to have immunomodulatory effects.

\section{RESULTS}

Of the patients who had an in-patient stay in $2000(n=142,169)$, $8,652(6.1 \%)$ subjects met the inclusion criteria. The mean \pm SD age was $75.2 \pm 6.1 \mathrm{yrs}, 54.5 \%$ of subjects were married and $98.6 \%$ were male. In this cohort, $13.5 \%$ were black, $78.7 \%$ were white, $5.3 \%$ were Hispanic and $2.5 \%$ were other/unknown. In the study cohort, $9.9 \%$ of the subjects died within 30 days of presentation and $17.2 \%$ died within 90 days of presentation.

TABLE 1 Characteristics of subjects ${ }^{\#}$ with pneumonia by use of statin and/or angiotensin-converting enzyme (ACE) inhibitors versus nonuse of either medication

\begin{tabular}{|c|c|c|c|}
\hline Variables & Use of statins and/or ACE inhibitors & Nonusers & p-value \\
\hline Subjects & 3728 & 4924 & \\
\hline Age yrs & $74.6 \pm 5.9$ & $75.7 \pm 6.3$ & $<0.001$ \\
\hline Males & 3669 (98) & $4858(99)$ & 0.4 \\
\hline \multicolumn{4}{|l|}{ Race } \\
\hline White & 2941 (79) & $3869(79)$ & \\
\hline Black & $491(13)$ & $680(14)$ & \\
\hline Hispanic & $206(6)$ & $256(5)$ & \\
\hline Other/unknown & $90(2)$ & $119(2)$ & 0.8 \\
\hline Married & $2181(59)$ & $2523(51)$ & $<0.001$ \\
\hline \multicolumn{4}{|l|}{ Charlson comorbid conditions } \\
\hline Myocardial infarction & $1115(30)$ & $687(14)$ & $<0.001$ \\
\hline $\mathrm{CHF}$ & $2184(59)$ & $1552(32)$ & $<0.001$ \\
\hline PVD & $1138(31)$ & $1013(21)$ & $<0.001$ \\
\hline Stroke & $1322(35)$ & $1356(28)$ & $<0.001$ \\
\hline Chronic lung disease & 2689 (72) & $3457(70)$ & 0.05 \\
\hline Peptic ulcer & $547(15)$ & $678(14)$ & 0.2 \\
\hline Rheumatologic disease & $202(5)$ & $273(6)$ & 0.8 \\
\hline Mild liver disease & $60(2)$ & $101(2)$ & 0.1 \\
\hline Diabetes & $1767(47)$ & $1268(26)$ & $<0.001$ \\
\hline Dementia & $351(9)$ & $663(14)$ & $<0.001$ \\
\hline Diabetes with complications & $797(21)$ & $417(8)$ & $<0.001$ \\
\hline Moderate liver disease & $18(0.5)$ & $34(1)$ & 0.2 \\
\hline Hemiplegia & $171(5)$ & $227(5)$ & 0.9 \\
\hline Renal disease & $485(13)$ & $429(9)$ & $<0.001$ \\
\hline Any malignancy & 1436 (39) & $2088(42)$ & $<0.001$ \\
\hline Metastatic solid tumour & $283(8)$ & $542(11)$ & $<0.001$ \\
\hline
\end{tabular}

Data are presented as $n$, mean \pm SD or $n(\%)$. CHF: congestive heart failure; PVD: peripheral vascular disease. ${ }^{\#}: n=8,652$. 


\begin{tabular}{|c|c|c|c|c|}
\hline TABLE 2 & $\begin{array}{l}\text { 30-day } \\
\text { converti } \\
\text { by age }\end{array}$ & ime & $\begin{array}{l}r \text { angi } \\
\text { hhibito }\end{array}$ & catified \\
\hline \multirow[t]{2}{*}{ Age yrs } & \multicolumn{2}{|c|}{ Statin use $\%$} & \multicolumn{2}{|c|}{ ACE inhibitor use \% } \\
\hline & Yes & No & Yes & No \\
\hline $65-74$ & 4.3 & 9.2 & 6.0 & 9.2 \\
\hline $75-84$ & 6.1 & 11.2 & 8.8 & 11.2 \\
\hline$>85$ & 5.4 & 20.0 & 15.4 & 20.5 \\
\hline
\end{tabular}

Table 1 shows the variables examined by use of statins and/or ACE inhibitors versus nonusers of either.

In the study cohort, $1,567(18.1 \%)$ subjects were receiving statins and 2,930 (33.9\%) were receiving ACE inhibitors. There were 2,161 subjects who were only receiving ACE inhibitors, 769 on both ACE inhibitors and statins, and 798 who were receiving only statins. In the bivariate analyses, both statin (10.9 versus $5.0 \%, p<0.001)$ and ACE inhibitor use (11 versus $7.8 \%, \mathrm{p}<0.0001)$ were both significantly associated with decreased 30-day mortality. There was a significant difference in both statin and ACE inhibitor use by age group, with younger subjects more likely to have received the medication(s) of interest. In total, $22.5 \%$ of subjects aged $65-74$ yrs, $15.5 \%$ aged $75-84$ yrs and $6.2 \%$ aged $>85$ yrs were receiving statins; while $35.6 \%$ of subjects aged $65-74$ yrs, $33.2 \%$ aged $75-$ $84 \mathrm{yrs}$ and $27 \%$ aged $>85$ yrs received ACE inhibitors. When the current authors stratified by age group, comparing use of the medications of interest and 30-day mortality, there was a survival benefit for those taking the medications of interest for each group (table 2).

Figures 1 and 2 were created using Cox proportional hazard models to estimate and chart the baseline survivor functions for statin use (fig. 1) and ACE inhibitor use (fig. 2) over the first 30 days after admission. Both statin and ACE inhibitor use were significantly associated with improved 30-day survival $(\mathrm{p}<0.0001)$.

In the generalised linear mixed-effect models, after adjusting for the appropriate propensity score and hospital admission, prior use of a statin (odds ratio (OR) 0.54, 95\% confidence interval (CI) 0.42-0.70) or ACE inhibitor (OR 0.80, 95\% CI 0.680.89) was significantly associated with decreased 30-day mortality. When the present authors included the variables used in the propensity score separately in the respective regression models, the OR (and CI) for statin or ACE inhibitors were very similar to previous results (statins: OR 0.57, 95\% CI 0.45-0.73; ACE inhibitor: OR 0.84, 95\% CI 0.72-0.98).

In addition, to examine whether there were interactions between statins and ACE inhibitors, the model was re-run with three medication variables (statin use only, ACE inhibitor use only, statins and ACE inhibitor use) and the individual potential confounders, rather than the propensity score. The current authors found that all three groups were associated with decreased mortality: ACE inhibitors alone (OR 0.84, 95\% CI $0.70-1.00)$; statins alone $(0.58,0.42-0.80)$; and those using both $(0.45,0.31-0.66)$.

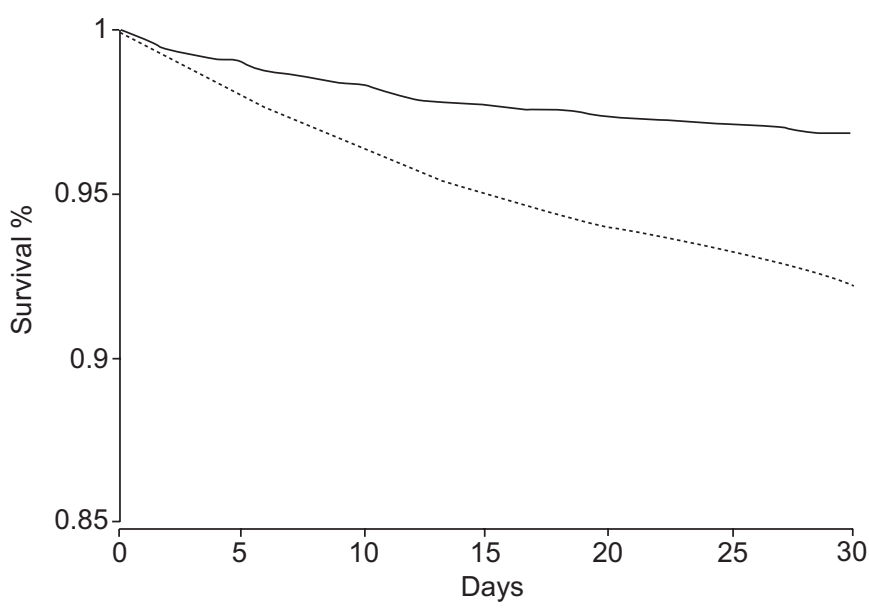

FIGURE 1. Proportion of surviving patients hospitalised with communityacquired pneumonia by statin use $(n=1,567 ;-)$ versus nonuse $(n=7,085 ; \cdots \cdots)$. $\mathrm{p}<0.0001$

Among the 5,379 subjects who were hospitalised during the influenza season, both of the generalised linear mixed-effect models were re-run and it was found that statin use was still significantly associated with decreased 30-day mortality (OR $0.52,95 \%$ CI 0.38-0.73), but ACE inhibitor (OR 0.92, 95\% CI $0.75-1.15)$ use was not significantly associated with mortality.

In the generalised linear mixed-effect model that examined nonstatin lipid-lowering medication use, there was no significant association with mortality (OR 0.97, 95\% CI 0.51-1.83).

\section{DISCUSSION}

The current authors found that prior outpatient use of statins and ACE inhibitors was associated with a decreased 30-day mortality for subjects aged $>65$ yrs hospitalised with CAP. This group is uniquely subject to increasing incidence of pneumonia and pneumonia-related mortality. In addition, it was found that,

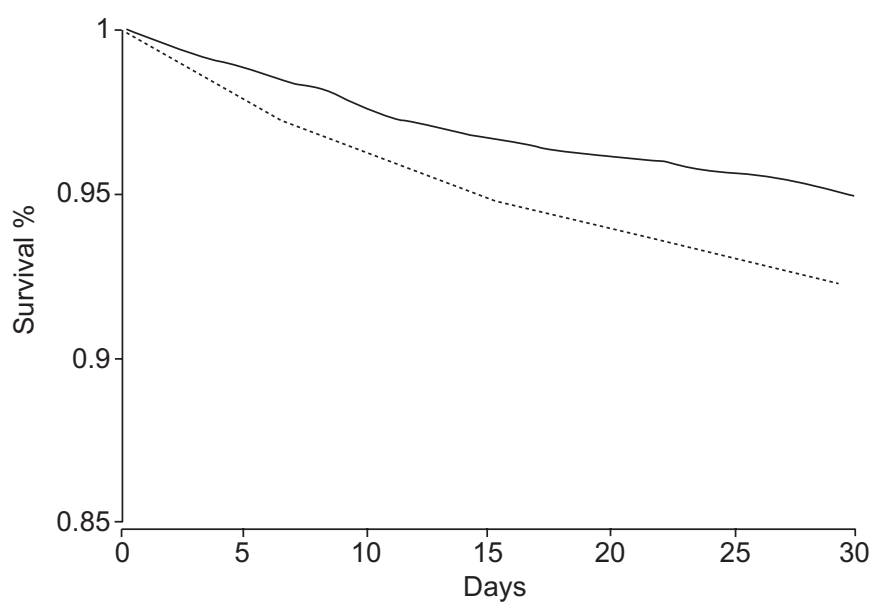

FIGURE 2. Proportion of surviving patients hospitalised with communityacquired pneumonia by use of angiotensin-converting enzyme inhibitor ( $n=2,930$; $\longrightarrow$ versus nonuse $(\mathrm{n}=5,722 ; \cdots) \cdot \mathrm{p}<0.0001$. 
when the analysis was restricted to subjects hospitalised with pneumonia during the influenza season, there continued to be decreased mortality for those who used statins. Further studies, including randomised controlled trials, are needed to examine the impact of statins and ACE inhibitors, both pre-hospitalisation and when initiated on acute presentation, on subjects hospitalised with CAP and influenza.

The present study supports the findings of recent studies, which demonstrated that subjects hospitalised with bacteraemia and pneumonia who were receiving statins at admission had a significant reduction in in-hospital or 30-day mortality [12-16]. In the study by LiAPPIS et al. [12], after adjustment for confounding factors (including comorbid conditions, age, concurrent medications, site of infection, vital signs and laboratory data), not receiving a statin (OR 7.6, 95\% CI 1.0157.5) was significantly associated with mortality. A previous retrospective cohort study of subjects hospitalised with CAP demonstrated that prior outpatient use of statins was associated with decreased pneumonia-related mortality (OR 0.36, 95\% CI 0.14-0.92) [14].

The present results do not support the recent study by MAJUMDAR et al. [19], which showed that statins were not associated with improved outcomes for patients with pneumonia. Although this study had a prospectively derived cohort with rich clinical data, there were several problems, which included a failure to assess interactions and multicollinearity in the face of counter-intuitive results. For example, the findings of MAJUMDAR et al. [19] that age $>65 \mathrm{yrs}$, ischaemic heart disease and using levofloxacin are protective against inhospital mortality and intensive care unit admission, or that pneumonia severity index [33] class III has an OR 2.45, have not been previously reported.

Regarding the use of ACE inhibitors, the present results suggest that, although they may be protective for some subjects with pneumonia, there does not appear to be a benefit for those who have pneumonia secondary to influenza. The protective effect seen in the present study and another [15] may be due to ACE inhibitors increasing serum levels of substance $P$, which is hypothesised to lead to a better gag reflex and increased clearance of secretions [37], rather than immunomodulatory effects [9-11]. In addition, ACE inhibitors have also been demonstrated to have pulmonary protective effects [38], and two recent studies [39, 40] demonstrated that a genetic polymorphism associated with increased activity of the reninangiotensin system is also associated with increased incidence of [39] or higher mortality from [40] acute respiratory distress syndrome. Although it appears that statins have a stronger effect upon pneumonia-related mortality, future research should still examine the impact of both classes of medications on infectious disease-related outcomes.

The present authors found that in the bivariate analyses, several variables, including younger age, being married and not having dementia or history of malignancy, were associated with use of statins or ACE inhibitors. These associations suggest the possible existence of a "healthy user" effect [41]. A strength of the current study was that the cohort had the same access to medical care and low-to-no-cost prescriptions, due to the structure of the VA healthcare system [42]. In addition, the current authors adjusted for these sociodemographic characteristics and comorbid conditions in the models. Future observational studies need to adjust for these and other potential characteristics that may impact the prescription and use of these medications.

Although the current study was a large database analysis and subject to the recognised limitations of such studies, the authors carefully assembled the cohort from complete patient discharge data to avoid ascertainment bias. The present sample was predominantly male dominated due to the authors' use of VA administrative data, and it is possible, but unlikely, that females may have differential responsiveness to statins compared with males. The present authors were also unable to assess factors such as in-patient continuation of the statins or the dose effect due to the lack of availability of these data. Further research is needed to examine these factors. Also, it was not possible to specifically examine the impact of statin use on subjects with influenza due to the infrequent testing for this condition and the fact that it is an infrequently coded discharge diagnosis [43]. However, since a protective association was found when those who were hospitalised with pneumonia during the influenza season were examined, it is possible that statins may be beneficial for those at high risk of death due to influenza. Finally, as in any nonexperimental study, the current authors were unable to state conclusively that the prior outpatient use of statins is the cause of decreased mortality in this cohort. However, since subjects receiving statins have numerous medical conditions significantly associated with increased short-term mortality, and the present analyses were adjusted for several factors associated with patient frailty or healthy user bias, the present authors feel that they have good evidence that these medications may have beneficial effects for subjects hospitalised with CAP.

In conclusion, the present study finds that prior outpatient use of statins and, to a lesser extent, angiotensin-converting enzyme inhibitors, is associated with lower mortality for subjects hospitalised with community-acquired pneumonia. These results suggest that there may be an additional benefit of statin and angiotensin-converting enzyme inhibitor use to the already compelling data for their use in subjects with vascular disease and diabetes. Randomised trials are needed to confirm the magnitude of the impact of statin and angiotensinconverting enzyme inhibitor use, either pre-hospitalisation or when initiated on acute presentation, on subjects hospitalised with community-acquired pneumonia and to elucidate the mechanism(s) by which they may work.

\section{REFERENCES}

1 Hoyert DL, Arias E, Smith BL, Murphy SL, Kochanek KD. Deaths: final data for 1999. Natl Vital Stat Rep 2001; 49: 1-113.

2 Fry AM, Shay DK, Holman RC, Curns AT, Anderson LJ. Trends in hospitalizations for pneumonia among persons aged 65 years or older in the United States, 1988-2002. JAMA 2005; 294: 2712-2719.

3 Bernard GR, Vincent JL, Laterre PF, et al. Efficacy and safety of recombinant human activated protein $\mathrm{C}$ for severe sepsis. N Engl J Med 2001; 344: 699-709. 
4 Moussa K, Michie HJ, Cree IA, et al. Phagocyte function and cytokine production in community acquired pneumonia. Thorax 1994; 49: 107-111.

5 Puren AJ, Feldman C, Savage N, Becker PJ, Smith C. Patterns of cytokine expression in community-acquired pneumonia. Chest 1995; 107: 1342-1349.

6 Bauer TT, Montón C, Torres A, et al. Comparison of systemic cytokine levels in patients with acute respiratory distress syndrome, severe pneumonia, and controls. Thorax 2000; 55: 46-52.

7 Jialal I, Stein D, Balis D, Grundy SM, Adams-Huet B, Devaraj S. Effect of hydroxymethyl glutaryl coenzyme a reductase inhibitor therapy on high sensitive C-reactive protein levels. Circulation 2001; 103: 1933-1935.

8 Strandberg TE, Vanhanen H, Tikkanen MJ. Effect of statins on C-reactive protein in patients with coronary artery disease. Lancet 1999; 353: 118-119.

9 Gullestad L, Aukrust P, Ueland T, et al. Effect of highversus low-dose angiotensin converting enzyme inhibition on cytokine levels in chronic heart failure. J Am Coll Cardiol 1999; 34: 2061-2067.

10 Alkharfy KM, Kellum JA, Matzke GR. Unintended immunomodulation: part II. Effects of pharmacological agents on cytokine activity. Shock 2000; 13: 346-360.

11 Skerrett SJ, Park DR. Anti-inflammatory treatment of acute and chronic pneumonia. Semin Respir Infect 2001; 16: $76-84$.

12 Liappis AP, Kan VL, Rochester CG, Simon GL. The effect of statins on mortality in patients with bacteremia. Clin Infect Dis 2001; 33: 1352-1357.

13 Kruger P, Fitzsimmons K, Cook D, Jones M, Nimmo G. Statin therapy is associated with fewer deaths in patients with bacteraemia. Intensive Care Med 2006; 32: 75-79.

14 Mortensen EM, Restrepo M, Anzueto A, Pugh J. The effect of prior statin use on 30-day mortality for patients hospitalized with community-acquired pneumonia. Respir Res 2005; 6: 82.

15 Mortensen EM, Restrepo MI, Anzueto A, Pugh J. The impact of prior outpatient ACE inhibitor use on 30-day mortality for patients hospitalized with communityacquired pneumonia. BMC Pulm Med 2005; 5: 12.

16 Almog Y, Shefer A, Novack V, et al. Prior statin therapy is associated with a decreased rate of severe sepsis. Circulation 2004; 110: 880-885.

17 Hackam DG, Mamdani M, Li P, Redelmeier DA. Statins and sepsis in patients with cardiovascular disease: a populationbased cohort analysis. Lancet 2006; 367: 413-418.

18 Fernandez R, De Pedro VJ, Artigas A. Statin therapy prior to ICU admission: protection against infection or a severity marker? Intensive Care Med 2006; 32: 160-164.

19 Majumdar SR, McAlister FA, Eurich DT, Padwal RS, Marrie TJ. Statins and outcomes in patients admitted to hospital with community acquired pneumonia: population based prospective cohort study. BMJ 2006; 333: 999.

20 Pugh MJ, Fincke BG, Bierman AS, et al. Potentially inappropriate prescribing in elderly veterans: are we using the wrong drug, wrong dose, or wrong duration? J Am Geriatr Soc 2005; 53: 1282-1289.

21 Marrie TJ, Durant H, Sealy E. Pneumonia - the quality of medical records data. Med Care 1987; 25: 20-24.
22 Whittle J, Fine MJ, Joyce DZ, et al. Community-acquired pneumonia: can it be defined with claims data? Am J Med Qual 1997; 12: 187-193.

23 Aronsky D, Haug PJ, Lagor C, Dean NC. Accuracy of administrative data for identifying patients with pneumonia. Am J Med Qual 2005; 20: 319-328.

24 Selim AJ, Berlowitz DR, Fincke G, et al. The health status of elderly veteran enrollees in the Veterans Health Administration. J Am Geriatr Soc 2004; 52: 1271-1276.

25 Charlson ME, Pompei P, Ales KL, MacKenzie CR. A new method of classifying prognostic comorbidity in longitudinal studies: development and validation. J Chron Dis 1987; 40: 373-383.

26 Charlson M, Szatrowski TP, Peterson J, Gold J. Validation of a combined comorbidity index. J Clin Epidemiol 1994; 47: $1245-1251$.

27 Schneeweiss S, Seeger JD, Maclure M, Wang PS, Avorn J, Glynn RJ. Performance of comorbidity scores to control for confounding in epidemiologic studies using claims data. Am J Epidemiol 2001; 154: 854-864.

28 Mortensen EM, Coley CM, Singer DE, et al. Causes of death for patients with community-acquired pneumonia: results from the Pneumonia Patient Outcomes Research Team cohort study. Arch Intern Med 2002; 162: 1059-1064.

29 Cowper DC, Kubal JD, Maynard C, Hynes DM. A primer and comparative review of major US mortality databases. Ann Epidemiol 2002; 12: 462-468.

30 Stone RA, Obrosky DS, Singer DE, Kapoor WN, Fine MJ. Propensity score adjustment for pretreatment differences between hospitalized and ambulatory patients with community-acquired pneumonia. Pneumonia Patient Outcomes Research Team (PORT) Investigators. Med Care 1995; 33: Suppl. 4, AS56-AS66.

31 Klungel $\mathrm{OH}$, Martens EP, Psaty BM, et al. Methods to assess intended effects of drug treatment in observational studies are reviewed. J Clin Epidemiol 2004; 57: 1223-1231.

32 Rosenbaum PR, Rubin DB. The central role of the propensity score in observational studies for causal effects. Biometrika 1983; 70: 41-55.

33 Fine MJ, Auble TE, Yealy DM, et al. A prediction rule to identify low-risk patients with community-acquired pneumonia. N Engl J Med 1997; 336: 243-250.

34 Mortensen EM, Cornell J, Whittle J. Racial variations in processes of care for patients with community-acquired pneumonia. BMC Health Serv Res 2004; 4: 20.

35 Rabe-Hesketh S, Skrondal A, Pickles A. Maximum likelihood estimation of limited and discrete dependent variable models with nested random effects. I Econom 2005; 128: 301-323.

36 Brammer TL, Murray EL, Fukuda K, Hall HE, Klimov A, Cox NJ. Surveillance for influenza - United States, 1997-98, 1998-99, and 1999-00 seasons. MMWR Surveill Summ 2002; 51: 1-10.

37 Nakayama K, Sekizawa K, Sasaki H. ACE inhibitor and swallowing reflex. Chest 1998; 113: 1425.

38 Mancini GB, Etminan M, Zhang B, Levesque LE, FitzGerald JM, Brophy JM. Reduction of morbidity and mortality by statins, angiotensin-converting enzyme inhibitors, and angiotensin receptor blockers in patients with chronic obstructive pulmonary disease. J Am Coll Cardiol 2006; 47: 2554-2560. 
39 Marshall RP, Webb S, Bellingan GJ, et al. Angiotensin converting enzyme insertion/deletion polymorphism is associated with susceptibility and outcome in acute respiratory distress syndrome. Am J Respir Crit Care Med 2002; 166: 646-650.

40 Jerng JS, Yu CJ, Wang HC, Chen KY, Cheng SL, Yang PC. Polymorphism of the angiotensin-converting enzyme gene affects the outcome of acute respiratory distress syndrome. Crit Care Med 2006; 34: 1001-1006.
41 Garbe E, Suissa S. Hormone replacement therapy and acute coronary outcomes: methodological issues between randomized and observational studies. Hum Reprod 2004; 19: 8-13.

42 Piette JD, Heisler M. Problems due to medication costs among VA and non-VA patients with chronic illnesses. Am J Manag Care 2004; 10: 861-868.

43 Doshi P. Are US flu death figures more PR than science? BMJ 2005; 331: 1412. 